\title{
New Schemes for Positive Real Truncation
}

\author{
Kari Unneland ${ }^{1}$ Paul Van Dooren ${ }^{2}$ Olav Egeland ${ }^{1}$
}

\author{
${ }^{1}$ Department of Engineering Cybernetics, Norwegian University of Science and Technology, N-7491 Trondheim, \\ Norway. E-mail: \{unneland, egeland\}@itk.ntnu.no \\ ${ }^{2}$ Department of Mathematical Engineering, Université Catholique de Louvain, B-1348 Louvian La Neuve, Belgium. \\ E-mail: \{vdooren\}@csam.ucl.ac.be
}

\begin{abstract}
Model reduction, based on balanced truncation, of stable and of positive real systems are considered. An overview over some of the already existing techniques are given: Lyapunov balancing and stochastic balancing, which includes Riccati balancing. A novel scheme for positive real balanced truncation is then proposed, which is a combination of the already existing Lyapunov balancing and Riccati balancing. Using Riccati balancing, the solution of two Riccati equations are needed to obtain positive real reduced order systems. For the suggested method, only one Lyapunov equation and one Riccati equation are solved in order to obtain positive real reduced order systems, which is less computationally demanding. Further it is shown, that in order to get positive real reduced order systems, only one Riccati equation needs to be solved. Finally, this is used to obtain positive real frequency weighted balanced truncation.
\end{abstract}

Keywords: Model Reduction, Balanced Truncation, Positive Realness, Frequency Weighting

\section{Introduction}

In simulation and control there is a need for efficient and compact mathematical models. Model reduction is a tool for reducing the size of high order mathematical models, where the focus is on approximating the most important dynamical features of the original model. Model order reduction is used in a wide variety of applications and different fields, such as very large-scale integration (VLSI) chip design, simulation of micro-electro-mechanical systems (MEMS), image processing, economical models, weather and air quality prediction and control design and synthesis.

In this paper an overview of already existing model reduction methods is given. We first focus on order reduction by balanced truncation, which is well suited and efficient for systems of moderate size (say, of an order of a few thousands). Recent results have shown that these type of algorithms also are promising for higher order systems, as better algorithms are being developed (Benner et al., 2005).
Positive real systems describes a class of systems which cannot generate energy internally; they can store and dissipate energy, but they cannot produce energy. For such systems, it is important that any approximate model reflects this property in order to avoid nonphysical behavior when used in numerical simulations. This is a useful property, which we would like to preserve in the model reduction process, and there are balanced truncation methods that indeed preserve the positive realness of a system during the reduction process. In this paper, we present a new algorithm in this class, which is computationally efficient.

In Section 2 we give an introduction to the most common balancing schemes, Lyapunov balancing and stochastic balancing. Based on these algorithms we propose a novel algorithm, which constructs positive real reduced order systems. A numerical example is given where the different schemes are compared in terms of computational efficiency and accuracy. In Section 3 we look at which properties are required in order to yield positive real reduced order systems. We then 
use this result to develop a method for positive real frequency weighted truncation.

\section{Balanced Truncation}

For an $n$th order minimal linear time invariant (LTI) system in state space form,

$$
\begin{aligned}
& \dot{x}=A x+B u, \\
& y=C x+D u,
\end{aligned}
$$

where $A \in \mathbb{R}^{n \times n}, B \in \mathbb{R}^{n \times m}, C \in \mathbb{R}^{p \times n}$ and $D \in$ $\mathbb{R}^{p \times m}$, the associated transfer function is given by

$$
G(s)=C\left(s I_{n}-A\right)^{-1} B+D .
$$

Model reduction deals with finding a reduced order system, with order $r \leq n$, which captures the main features of the original system, i.e. its dynamics, system stability, system passivity and possibly some structural properties. We denote the reduced model of order $r \leq n$ by

$$
\begin{aligned}
\dot{x}_{r} & =A_{r} x_{r}+B_{r} u, \\
y & =C_{r} x_{r}+D_{r} u,
\end{aligned}
$$

where $A_{r} \in \mathbb{R}^{r \times r}, B_{r} \in \mathbb{R}^{r \times m}, C_{r} \in \mathbb{R}^{p \times r}$ and $D_{r} \in$ $\mathbb{R}^{p \times m}$, and its associated transfer function is given by

$$
G_{r}(s)=C_{r}\left(s I_{r}-A_{r}\right)^{-1} B_{r}+D_{r}
$$

Many different coordinate systems can be used to describe the dynamical system in (1). Let $T \in \mathbb{R}^{n \times n}$ be a nonsingular matrix, and let the system undergo a state space transformation,

$$
\begin{aligned}
\bar{x} & =T x, \\
\dot{\bar{x}} & =T A T^{-1} \bar{x}+T B u, \\
y & =C T^{-1}+D u .
\end{aligned}
$$

This transformed system (5-6) has the same dynamics for any nonsingular matrix $T$. Model reduction can now be done by choosing $T$, in terms of some physical measure, and discard the parts of the transformed state $\bar{x}$ which are less important in terms of that measure. Partitioning the matrices $T A T^{-1}, T B, C T^{-1}$ accordingly,

$$
\begin{aligned}
T A T^{-1} & =\left[\begin{array}{ll}
\bar{A}_{11} & \bar{A}_{12} \\
\bar{A}_{21} & \bar{A}_{22}
\end{array}\right], \\
T B & =\left[\begin{array}{l}
\bar{B}_{1} \\
\bar{B}_{2}
\end{array}\right], \\
C T^{-1} & =\left[\begin{array}{ll}
\bar{C}_{1} & \bar{C}_{2}
\end{array}\right],
\end{aligned}
$$

the reduced order system can thus be written as,

$$
A_{r}=\bar{A}_{11}, B_{r}=\bar{B}_{1}, C_{r}=\bar{C}_{1}, D_{r}=D .
$$

This procedure of reducing systems is called projection based model order reduction (PBMOR). Depending on which properties the PBMOR keeps (e.g. stability or passivity) or on which $T$ is chosen, different classes of PBMOR are obtained.

One important class of PBMOR method is balanced truncation, where the coordinate transformation $T$ in (4)-(6) is chosen such that two given positive definite matrices (say, $M$ and $N$ ) are transformed via $\bar{M}:=T M T^{T}, \bar{N}:=T^{-T} N\left(T^{-1}\right)$ to become equal and diagonal :

$$
\bar{M}=\bar{N}=\Sigma .
$$

The resulting transformed system is then in a so-called balanced coordinate system based on $M$ and $N$. For instance, in order to obtain asymptotically stable systems one chooses $M$ and $N$ as the solution of a Lyapunov equation; for positive real systems one chooses them as a solution of the positive real lemma. The matrices $M$ and $N$ are then clearly related to the properties of the system (i.e. the Lyapunov equations, the positive real equations) and will lead to different types of balancing and hence also to different types of reduced order models. The idea of balanced systems were introduced by (Mullis and Roberts, 1976) in digital filters, and later introduced to the system and control society by (Moore, 1981). An nice survey of balanced truncation methods is given in (Gugercin and Antoulas, 2004).

\subsection{Lyapunov Balancing}

Lyapunov balanced truncation was introduced to the systems and control society by (Moore, 1981). It is based on the solution of two Lyapunov equations, defining the controllability gramian $P$, and the observability gramian $Q$ :

$$
\begin{aligned}
& A P+P A^{T}+B B^{T}=0, \\
& A^{T} Q+Q A+C^{T} C=0 .
\end{aligned}
$$

Notice that the gramians are positive definite if the system is minimal. The idea behind the Lyapunov balancing is to transform the mathematical model to a coordinate system where the states that are difficult to control are also hard to observe. The reduced model is obtained by discarding the states which have this property. Below we recall an algorithm for finding transformations $T$ and $T^{-1}$ satisfying (4)-(6). Lyapunov balanced truncation then amounts to using the positive definite controllability and observability gramians $(P, Q)$ for the matrices $(M, N)$. 


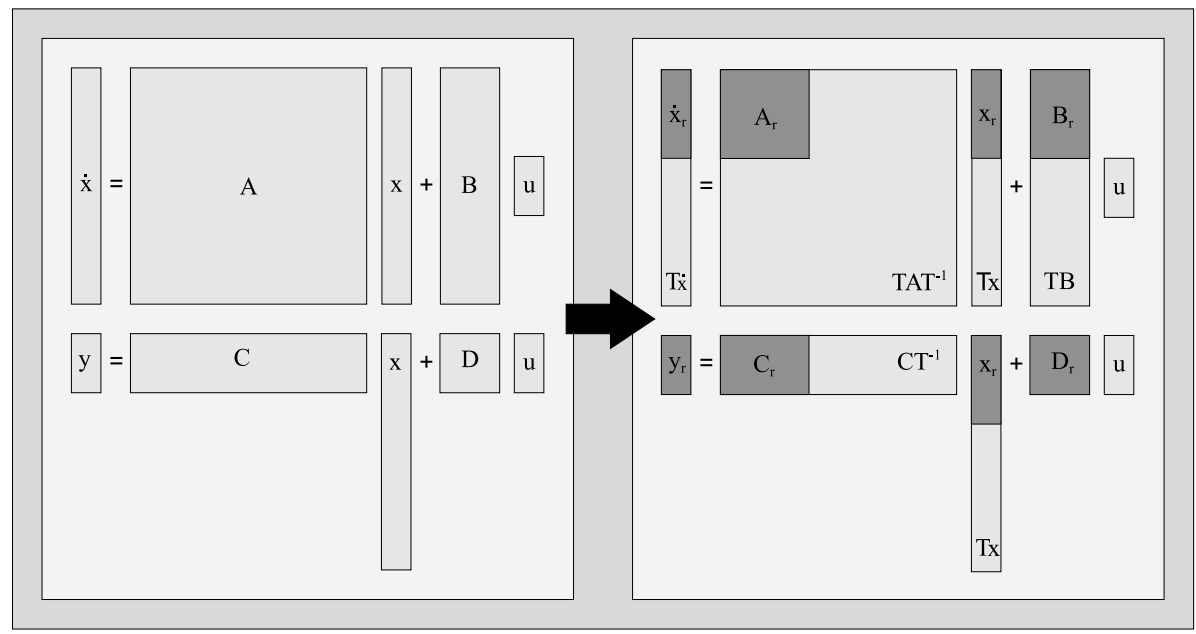

Figure 1: Order reduction by projection

Table 1: Balanced Truncation Algorithm

1. Choose a pair of positive definite matrices $(M, N)$

2. Compute the Cholesky factorizations of $M$ and $N$ $M=L_{M} L_{M}^{T}, \quad N=L_{N} L_{N}^{T}$

3. Compute the SVD of $L_{M}^{T} L_{N}$

$$
L_{M}^{T} L_{N}=U \Sigma V^{T}
$$

4. Construct the balancing transformations $T=\Sigma^{1 / 2} U^{T} L_{M}^{-1}, T^{-1}=L_{N}^{-T} V \Sigma^{1 / 2}$

5. Construct the balanced realization $\bar{A}=T A T^{-1}, \bar{B}=T B, \bar{C}=C T^{-1}$, yielding $T M T^{T}=T^{-T} N T^{-1}=\Sigma$

6. Truncate $\bar{A}, \bar{B}, \bar{C}$ to form the reduced order system $A_{r}, B_{r}, C_{r}$

The physical interpretation of the Lyapunov balancing can be related to the $L_{2}$-norm of the input and the output of the system. The controllability and observability gramians $P$ and $Q$ are related to the energy demanded to control and observe the system (Glover, 1984). The controllability gramian $P$ is connected to the solution of the minimum $L_{2}$-norm problem,

$\min _{u \in L_{2}(-\infty, 0)}\left\{\int_{-\infty}^{0} u(t)^{T} u(t) d t\right.$ s.t. $\left.x(0)=x_{0}\right\}=x_{0}^{T} P^{-1} x_{0}$.

In this setting the size of the eigenvalues of $P$ describes (in the $L_{2}$-norm) how much input energy is needed to control the associated state eigenvector. The observability gramian $Q$ is related to the $L_{2}$-norm of the output. If the system is released at $x(0)=x_{0}$ with $u(t)=0, \forall t \geq 0$ the following equality holds,

$$
\int_{0}^{\infty} y(t)^{T} y(t) d t=x_{0}^{T} Q x_{0}
$$

In this setting, the size of the eigenvalues of $Q$ describes (in the $L_{2}$-norm) how much output energy is produced when the associated state eigenvector is in free evolution.

The balanced systems based on the positive definite matrices $(P, Q)$,

$$
\bar{A}=T A T^{-1}, \bar{B}=T B, \bar{C}=C T^{-1},
$$

where,

$$
T P T^{T}=T^{-T} Q T^{-1}=\Sigma,
$$

are now in a coordinate system where the observability and controllability gramians are equal and diagonal. Here $\Sigma$ represents the singular values of the system

$$
\Sigma=\operatorname{diag}\left(\sigma_{1} \geq \sigma_{2} \geq \ldots \geq \sigma_{n}\right) .
$$

Since the system is in a balanced coordinate system, the singular values give a measure of which states are difficult to control and observe, and can therefore be discarded without affecting too much the input-output behaviour. Hence, looking at the singular values of a system provides a good way to measure which states to keep and which ones to discard.

An attractive part of the Lyapunov balancing is that there exists a well defined error bound between the original and reduced order system (Glover, 1984),

$$
\left\|G(s)-G_{r}(s)\right\|_{\infty} \leq \sum_{k=r+1}^{n} \sigma_{k}
$$


where $n$ is the order of the original system and $r$ is the order of the reduced order system.

When applied to an asymptotically stable system, Lyapunov balancing preserves the stability of the system, but a property like passivity might not be preserved. We will call a state transformation $T$ which guarantees that the reduced order system is stable as well, a stable state space transformation.

Definition 1 Let $G(s)$ in (3) be a stable minimal realization, then $T \in \mathbb{R}^{n \times n}$ is called a stable state transformation if all the truncated systems,

$$
G_{r}(s)=C_{r}\left(s I-A_{r}\right)^{-1} B_{r}+D_{r}, r=(1, \ldots, n-1),
$$

obtained from the transformed system (4)-(6) are stable.

\subsection{Stochastic Balancing}

Stochastic balancing was first proposed by (Desai and Pal, 1984) where it was used to balance stochastic systems; (Harshavardhana et al., 1984) then showed that it preserves the positive realness of the original system. In (Green, 1988) it it shown how this can be applied to LTI systems. The idea behind stochastic balancing leads to three different model order reduction algorithms, as will be shown in this section.

Let $\Phi$ be the power spectrum of the positive real minimal degree transfer function $Z(s)=H\left(s I_{n}-F\right)^{-1} G+$ $J$, then we have the following relation (Obinata and Anderson, 2001)

$$
\Phi=Z(s)+Z^{T}(-s)=V(s) V^{T}(-s)=W^{T}(-s) W(s) .
$$

Here $Z(s)$ denotes the phase system, $V(s)$ the left spectral factor of $Z(s)$, and $W(s)$ the right spectral factor of $Z(s)$. The system $Z(s)=(F, G, H, J)$, which is positive real $(\mathrm{PR})$, satisfies the positive real lemma equations,

$$
\begin{gathered}
F R+R F^{T}=-G_{l} G_{l}^{T}, \\
R H^{T}-G=-G_{l} J_{l}^{T}, \\
-J-J^{T}=-J_{l} J_{l}^{T} .
\end{gathered}
$$

Here $R=R^{T}>0, G_{l}$ and $J_{l}$ can be solved from these equations. A dual pair of positive real equations can be obtained by pre- and post-multiplying (8) by $R^{-1}$, and pre-multiplying (9) by $R^{-1}$. By defining;

$$
\begin{aligned}
O & :=R^{-1}, \\
H_{r} & :=-L^{T} G_{l}^{T} R^{-1}, \\
J_{r} & :=L^{T} J_{l}^{T},
\end{aligned}
$$

where $L$ is an arbitrary orthogonal matrix (i.e. $L L^{T}=$ $I$ ), we obtain dual positive real (DPR) equations, given by

$$
\begin{aligned}
F^{T} O+O F & =-H_{r}^{T} H_{r} \\
O G-H^{T} & =-H_{r}^{T} J_{r} \\
-J-J^{T} & =-J_{r}^{T} J_{r} .
\end{aligned}
$$

Here $O=O^{T}>0, H_{r}$ and $J_{r}$ can be solved from these equations, which show that the dual system $Z^{T}(-s)=$ $G^{T}\left(s I-F^{T}\right)^{-1} H^{T}+J^{T}$ of $Z(s)$ is positive real.

The solutions $R$ and $O$ of (8)-(10) and (11)-(13) form convex sets (Willems, 1971)

$$
\begin{aligned}
& 0<R_{\min } \leq R \leq R_{\max } \\
& 0<O_{\min } \leq O \leq O_{\max }
\end{aligned}
$$

Given the solution $R$ to the PR equations, then $O=$ $R^{-1}$ is a solution to the DPR equations, hence $R_{\text {min }}=$ $O_{\max }^{-1}$ and $O_{\min }=R_{\max }^{-1}$.

Let $\left(R, G_{l}, J_{l}\right)$ be the solution to the PR equations (8)-(10), then the left spectral factor associated with $\left(R, H_{l}, J_{l}\right)$ is

$$
V(s)=H(s I-F)^{-1} G_{l}+J_{l} .
$$

Let $\left(O, H_{r}, J_{r}\right)$ be the solution to the DPR equations in (11)-(13), then the right spectral factor associated with $\left(O, H_{r}, J_{r}\right)$ is

$$
W(s)=H_{r}(s I-F)^{-1} G+J_{r} .
$$

A function $F_{c}$ can now be defined using $F, G_{l}$ and $H_{r}$ from (8) and (11) such that (Obinata and Anderson, 2001),

$$
\left[\begin{array}{cc}
Z(s) & V(s) \\
W(s) & F_{c}(s)
\end{array}\right]=\left[\begin{array}{c}
H \\
H_{r}
\end{array}\right](s I-F)^{-1}\left[\begin{array}{ll}
G & G_{l}
\end{array}\right]+\left[\begin{array}{cc}
J & J_{l} \\
J_{h} & 0
\end{array}\right] .
$$

By doing balancing truncation on $F_{c}(s)=H_{r}(s I-$ $F)^{-1} G_{l}$ based on its controllability gramian and observability gramian,

$$
\begin{gathered}
F P+P F^{T}+G_{l} G_{l}^{T}=0, \\
F^{T} Q+Q F+H_{r}^{T} H_{r}=0,
\end{gathered}
$$

induced truncations of the realizations $Z(s), V(s)$ and $W(s)$ are simultaneously obtained. Depending on whether you choose the system $G(s)$, which is to be reduced, equal to $Z(s), V(s)$ or $W(s)$ one ends up with three different order reduction algorithms; Riccati balancing (phase system balancing), left spectral factor balancing and right spectral factor balancing. 


\section{Riccati balancing (phase system balancing)}

By choosing the phase system equal to the system transfer function, $Z(s)=G(s)$, one obtains Riccati balancing, also called phase system balancing. It is now assumed that $G(s)=(A, B, C, D)$ is a minimal positive real transfer function, hence the system will satisfy the PR equations (8)-(10) and the DPR equations (11)-(13).

In Riccati balancing the minimal solution $\left(R_{m i n}, O_{\min }\right)$ to (8)-(10) and (11)-(13) are used. These can be obtained by rewriting (8)-(10) and (11)-(13) as a dual pair of Riccati equations, and then solve for $R>0$ and $O>0$;

$$
\begin{aligned}
& F R+R F^{T}+\left(R H^{T}-G\right)\left(J+J^{T}\right)^{-1}\left(H R-G^{T}\right)=0 \\
& F^{T} O+O F+\left(O G-H^{T}\right)\left(J+J^{T}\right)^{-1}\left(G^{T} O-H\right)=0 .
\end{aligned}
$$

When $Z(s)=G(s)$ this gives,

$$
\begin{gathered}
A R+R A^{T}+\left(R C^{T}-B\right)\left(D+D^{T}\right)^{-1}\left(C R-B^{T}\right)=0, \\
A^{T} O+O A+\left(O B-C^{T}\right)\left(D+D^{T}\right)^{-1}\left(B^{T} O-C\right)=0 .
\end{gathered}
$$

By performing Riccati balancing the system is transformed to a basis where,

$$
R=O=\Sigma
$$

Since these are the minimal solutions to (8)-(10) and (11)-(13) (Antoulas, 2005),

$$
R_{\text {min }}=O_{\text {min }}=\Sigma \text {. }
$$

In (Green, 1988) it is shown that since the minimal solutions $R_{\min }$ and $O_{\min }$ are balanced, $O_{\min }^{-1}=R_{\max } \geq$ $R_{\text {min }}$ the $\sigma_{i}$ in (17) are all less than or equal to 1 , hence for Riccati balancing,

$$
\Sigma \leq I
$$

When applied to positive real systems, this property is preserved in the reduction process. We will call such state transformation $T$ given by the balancing of $(R, O)$ a positive real state transformation,

Definition 2 Let $G(s)$ in (3) be a positive real minimal realization, then $T \in \mathbb{R}^{n \times n}$ is called a positive real state transformation if all the truncated systems,

$$
G_{r}(s)=C_{r}\left(s I-A_{r}\right)^{-1} B_{r}+D_{r}, r=(1, \ldots, n-1),
$$

obtained from the transformed system (4)-(6) are positive real.

Like the Lyapunov balancing this also has a physical interpretation. Another way of checking if a system is passive, is in terms of Lyapunov theory and the use of storage functions (Willems, 1971). In these terms a system is said to be passive if there exists a storage function, $V(x)>0$, such that the following inequality holds

$$
V(x) \leq V(x(0))+\int_{0}^{t} s(u(t), y(t)) d t .
$$

Here $s(u(t), y(t))$ is called the supply function, and describes the rate at which power is supplied to the system. Two quantities can be defined from the notion of a storage function (Willems, 1971): the required supply, $V_{r}$, and the available storage, $V_{a}$. The required supply, $V_{r}$, is defined as

$$
0 \leq V_{r}\left(x_{0}\right)=\inf _{u(t) \mid x(0)=x_{0}}\left[\int_{-\infty}^{0} s(u(t), y(t)) d t\right],
$$

and it is the minimum amount of energy that must be injected in the system in order to control the system to state $x_{0}$ at time 0 . The solution of (15) is related to the required supply (Phillips et al., 2003)

$$
x_{0}^{T} R^{-1} x_{0}=V_{r}\left(x_{0}\right)
$$

In this setting, the size of the eigenvalues of $R$ describes how much energy is needed to control the associated state eigenvector. Small eigenvalues of $R$ imply that a large amount of energy is needed to reach the associated mode. $R$ can be looked on as an input energy gramian; we will refer to $R$ as the required supply gramian.

The available storage is defined as

$$
0 \leq V_{a}\left(x_{0}\right)=\sup _{x(0)=x_{0}}-\left[\int_{0}^{\infty} s(u(t), y(t)) d t\right],
$$

it is the maximum amount of energy which can be extracted from the system in free evolution. The solution of (16) is related to the available storage

$$
x_{0}^{T} O x_{0}=V_{a}\left(x_{0}\right) .
$$

Here, the size of the eigenvalues of $O$ describes how much energy can be extracted from the system in free evolution. Small eigenvalues of $O$ imply that a small amount of energy can be extracted from the associated mode. $O$ can be interpreted as an output energy gramian; we will refer to $O$ as the available storage gramian.

By doing Riccati balancing, the system is balanced in terms of its required supply and available storage. States which are associated with small amount of available storage and large amounts of required supply will be discarded. This balancing scheme is commonly used to reduce positive real systems, and will preserve this property in the reduction process. 


\section{Left spectral factor balancing}

Having the relation (7),

$$
Z(s)+Z^{T}(-s)=V(s) V^{T}(-s),
$$

the left spectral factor $V(s)$ can be found from the solution, $\left(R, G_{l}, J_{l}\right)$, to the PR equations (8)-(10),

$$
V(s)=H(s I-F)^{-1} G_{l}+J_{l} .
$$

Let the left spectral factor be the transfer function to be reduced, $G(s):=V(s)$ :

$$
V(s)=G(s)=H(s I-F)^{-1} G_{l}+J_{l}=C(s I-A)^{-1} B+D .
$$

Then there exists a positive real function $Z(s)$,

$$
Z(s)=H(s I-F)^{-1} G+J=C(s I-A)^{-1} G+J
$$

which is connected to $V(s)$ through the PR equations in (8)-(10). They can now be written as,

$$
\begin{aligned}
A R+R A^{T} & =-B B^{T}, \\
R C^{T}-G & =-B D^{T}, \\
-J-J^{T} & =-D D^{T} .
\end{aligned}
$$

The controllability gramian $R$ of the left spectral factor can be solved from (20), hence it is the same as the required supply gramian, $R$, of the positive real function $Z(s)$. By first solving for $R$ in (20), $G$ in (21) can be solved for,

$$
G=R C^{T}+B D^{T}
$$

The dual positive real equations for $Z(s)$ in (19) are now,

$$
\begin{aligned}
A^{T} O+O A & =-H_{r}^{T} H_{r}, \\
O G-C^{T} & =-H_{r}^{T} J_{r}, \\
-J-J^{T} & =-J_{r} J_{r}^{T} .
\end{aligned}
$$

Let this be rewritten as a Riccati equation, where $J+$ $J^{T}$ has been substituted for $D D^{T}$ in (22)

$$
A^{T} O+O A+\left(O G-C^{T}\right)\left(D D^{T}\right)^{-1}\left(G^{T} O-C\right)=0 .
$$

By balancing $G(s)$ based on the solution matrices $(R, O)$, left spectral balancing can be obtained. Given $G(s)$, the equations which needs to be solved are (20),(23) and (24).

\section{Right spectral factor balancing}

As for the left spectral factor, when the relation (7) is given,

$$
Z(s)+Z^{T}(-s)=W^{T}(-s) W(s)
$$

the right spectral factor $W(s)$ can be found from the solution $\left(O, H_{r}, J_{r}\right)$ to the DPR equations (11)-(13),

$$
W(s)=H_{r}(s I-F)^{-1} G+J_{r} .
$$

Let the right spectral factor be set equal to the transfer function of the system $W(s)=G(s)$,

$$
W(s)=G(s)=H_{r}(s I-F)^{-1} G+J_{r}=C(s I-A)^{-1} B+D .
$$

Now there exists a positive real function $Z(s)$,

$$
Z(s)=H(s I-F)^{-1} G+J=H(s I-A)^{-1} B+J,
$$

which is connected to $W(s)$ through the DPR equations in (11)-(13). They can now be written as,

$$
\begin{aligned}
A^{T} O+O A & =-C C^{T}, \\
O B-H^{T} & =-C^{T} D, \\
-J-J^{T} & =-D^{T} D .
\end{aligned}
$$

The observability gramian $O$ of the right spectral factor can be solved from (26), hence it is the same as the available storage gramian $O$ of the positive real function $Z(s)$. By first solving for $O$ in $(26), H$ in $(27)$ can be solved for,

$$
H^{T}=O B+C^{T} D
$$

The positive real equations for $Z(s)$ in $(25)$ are now,

$$
\begin{aligned}
A R+R A^{T} & =-G_{l} G_{l}^{T}, \\
R H^{T}-B & =-G_{l} J_{l}^{T}, \\
-J-J^{T} & =-J_{l} J_{l}^{T} .
\end{aligned}
$$

Let this be rewritten as a Riccati equation where $J+J^{T}$ has been substituted for $D^{T} D$ in (28),

$$
A R+R A^{T}+\left(R H^{T}-B\right)\left(D^{T} D\right)^{-1}\left(H R-B^{T}\right)=0 .
$$

By balancing $G(s)$ based on the solution matrices $(R, O)$, right spectral balancing can be obtained. Given $\mathrm{G}(\mathrm{s})$, the equations which needs to be solved are (26), (29) and (30). In Table 3 an overview over the different balancing schemes induced by balancing $F_{c}$ in (14) is given.

\section{Equality of the balancing schemes}

For SISO systems, the reduced order systems generated by the left spectral factor balancing and the right spectral factor balancing will have the same transfer function. Two systems state space representations are said to be zero-state equivalent if they have the same transfer matrix (Chen, 1999), 
Unneland, Van Dooren., "New Schemes for Positive Real Truncation"

Table 2: Overview over the different balancing schemes and associated equations to be solved

\begin{tabular}{|c|c|c|c|}
\hline \multicolumn{4}{|c|}{$\Phi(s)=V(s) V^{T}(-s)=Z(s)+Z^{T}(-s)=W^{T}(-s) W(s)$} \\
\hline $\begin{array}{l}\text { Balancing } \\
\text { Method }\end{array}$ & $\begin{array}{c}\text { Left Spectral Factor } \\
V(s)=H(s I-F)^{-1} G_{l}+J_{l}\end{array}$ & $\begin{array}{c}\text { Phase System } \\
Z(s)=H(s I-F)^{-1} G+J\end{array}$ & $\begin{array}{c}\text { Right Spectral Factor } \\
W(s)=H_{r}(s I-F)^{-1} G+J_{r}\end{array}$ \\
\hline$\Downarrow$ & $V(s)=C(s I-A)^{-1} G_{l}+J_{l}$ & $\begin{aligned} G(s)= & (A, B, C, D) \text { given } \\
& Z(s)=G(s) \\
Z(s)= & C(s I-A)^{-1} B+D\end{aligned}$ & $W(s)=H_{r}(s I-A)^{-1} B+J_{r}$ \\
\hline $\begin{array}{l}\text { Lyapunov bal.; } \\
\text {-solve for }(P, Q) \\
\text {-balance }(P, Q)\end{array}$ & & $\begin{array}{c}\text { Controllability gramian, } P: \\
A P+P A^{T}+B B^{T}=0 \\
\times \\
\text { Observability gramian, } Q: \\
A^{T} Q+Q A+C^{T} C=0\end{array}$ & \\
\hline $\begin{array}{l}\text { Riccati bal.; } \\
\text {-solve for }(R, O) \\
\text {-balance }(R, O)\end{array}$ & $\begin{array}{c}\text { Contr. gramian, } R: \Leftarrow \\
A R+R A^{T}+G_{l} G_{l}^{T}=0\end{array}$ & $\begin{array}{c}\Rightarrow \text { Required supply gramian, } R: \\
A R+R A^{T}=-G_{l} G_{l}^{T} \\
R C^{T}-B=-G_{l} J_{l}^{T} \\
-D-D^{T}=-J_{l} J_{l}^{T} \\
\mathbb{1} \\
\times \\
A R+R A^{T}+\left(R C^{T}-B\right)\left(D+D^{T}\right)^{-1}\left(C R-B^{T}\right)=0 \\
\text { Available storage gramian, } O: \Leftarrow \\
A^{T} O+O A=-H_{r}^{T} H_{r} \\
O B-C^{T}=-H_{r}^{T} J_{r} \\
-D-D^{T}=-J_{r}^{T} J_{r} \\
\mathbb{\mathbb { N }} \\
A^{T} O+O A+\left(O B-C^{T}\right)\left(D+D^{T}\right)^{-1}\left(B^{T} O-C\right)=0\end{array}$ & $\begin{array}{l}\Rightarrow \text { Obs. gramian, } O: \\
A^{T} O+O A+H_{r}^{T} H_{r}\end{array}$ \\
\hline \multirow[t]{2}{*}{$\begin{array}{l}\text { Mixed bal.; } \\
\text {-solve for } \\
\quad(P, O) \text { or }(R, Q) \\
\text {-balance } \\
(P, O) \text { or }(R, Q)\end{array}$} & $\begin{array}{c}\text { Contr. gramian, } R: \Leftarrow \\
A R+R A^{T}+G_{l} G_{l}^{T}=0\end{array}$ & $\begin{array}{c}\text { Controllability gramian, } P: \\
A P+P A^{T}+B B^{T}=0 \\
\times \quad \\
\text { Available storage gramian, } O: \Leftarrow \\
A^{T} O+O A+\left(O B-C^{T}\right)\left(D+D^{T}\right)^{-1}\left(B^{T} O-C\right)=0 \\
\text { or } \\
\Rightarrow \text { Required supply gramian, } R: \\
A R+R A^{T}+\left(R C^{T}-B\right)\left(D+D^{T}\right)^{-1}\left(C R-B^{T}\right)=0 \\
\times \\
\text { Observability gramian, } Q: \\
A^{T} Q+Q A+C^{T} C=0\end{array}$ & $\begin{array}{l}\Rightarrow \text { Obs. gramian, } O: \\
A^{T} O+O A+H_{r}^{T} H_{r}\end{array}$ \\
\hline & $\begin{array}{c}G(s)=(A, B, C, D) \text { given } \\
V(s)=G(s) \\
V(s)=C(s I-A)^{-1} B+D\end{array}$ & $Z(s)=C(s I-A)^{-1} G+J$ & $W(s)=H_{r}(s I-A)^{-1} G+J_{r}$ \\
\hline \multirow[t]{2}{*}{$\begin{array}{l}\text { Left spectral } \\
\text { factor bal.; } \\
\text {-solve for }(R, G, O) \\
\text {-balance }(R, O)\end{array}$} & $\begin{array}{l}\text { Contr. gramian, } R: \Leftarrow \\
A R+R A^{T}+B B^{T}=0\end{array}$ & $\begin{array}{c}\Rightarrow \text { Required supply gramian, } R: \\
A R+R A^{T}=-B B^{T} \\
G=R C^{T}+B D^{T} \\
-J-J^{T}=-D D^{T} \\
\times \\
\text { Available storage gramian, } O: \Leftarrow \\
A^{T} O+O A+\left(O G-C^{T}\right)\left(D D^{T}\right)^{-1}\left(G^{T} O-C\right)=0\end{array}$ & $\begin{array}{c}\Rightarrow \text { Obs. gramian, } O \\
A^{T} O+O A+H_{r}^{T} H_{r}\end{array}$ \\
\hline & $V(s)=H(s I-A)^{-1} G_{l}+J_{l}$ & $Z(s)=H(s I-A)^{-1} B+J$ & $\begin{array}{c}G(s)=(A, B, C, D) \text { given } \\
W(s)=G(s) \\
W(s)=C(s I-A)^{-1} B+D\end{array}$ \\
\hline $\begin{array}{l}\text { Right spectral } \\
\text { factor bal.: } \\
\text {-solve for }(O, H, R) \\
\text {-balance }(R, O)\end{array}$ & & $\begin{array}{c}\text { Available storage gramian, } O: \Rightarrow \\
A^{T} O+O A=-C^{T} C \\
H^{T}=O B+C^{T} D \\
-J-J^{T}=-D^{T} D \\
\times \\
\text { Required supply gramian, } R: \\
A R+R A^{T}+\left(R H^{T}-B\right)\left(D^{T} D\right)^{-1}\left(H R-B^{T}\right)=0\end{array}$ & $\begin{array}{l}\Leftarrow \text { Obs. gramian, } O: \\
A^{T} O+O A=-C^{T} C\end{array}$ \\
\hline
\end{tabular}


Theorem 1 Two linear time-invariant state equations $[A, B, C, D]$ and $[\bar{A}, \bar{B}, \bar{C}, \bar{D}]$ are zero-state equivalent or have the same transfer matrix if $D=\bar{D}$ and

$$
C A^{m} B=\bar{C} \bar{A}^{m} \bar{B}, \quad m=0,1,2, \ldots
$$

For SISO systems the transfer function $G(s)=G^{T}(s)$. Hence, for SISO systems, the dual system $G^{T}(s)$ is zero-state equivalent with the given system $G(s)$.

Proposition 1 The reduced order systems $G_{r}(s)$ given by left spectral factor balancing and right spectral factor balancing of the system $G(s)$ are zero-state equivalent.

Proof. Let us substitute the system $G(s)$ with the dual system $G^{T}(s)$ in (18),

$$
V(s)=G^{T}(s)=B^{T}\left(s I-A^{T}\right) C^{T}+D^{T} .
$$

Left spectral factor balancing of the dual system $G^{T}(s)$ gives the following equations,

$$
\begin{gathered}
A^{T} R+R A=-C^{T} C, \\
G=R B+C^{T} D, \\
-J-J^{T}=D^{T} D \\
A O+O A^{T}-\left(O G-B^{T}\right)\left(D^{T} D\right)^{-1}\left(G^{T} O-B^{T}\right)=0 .
\end{gathered}
$$

Looking at Table 3, one can see that left spectral factor balancing of the dual system $G^{T}(s)$ is the same as right spectral factor balancing of the system $G(s)$. The same yields for right spectral factor balancing of the dual system $G^{T}(s)$, which gives the same equations as for left spectral factor balancing of $G(s)$. Due to this duality the reduced order systems $G_{r}(s)$ given by left spectral factor balancing or right spectral factor balancing of $G(s)$ will be zero-state equivalent.

There is no similar physical interpretation to the left and right spectral factor balancing schemes, as for the Lyapunov and Riccati balancing schemes. But in (Opdenacker and Jonckheere, 1986) it is shown that these schemes give reduced spectral factors such that their phases approximate the phases of the original spectral factors. Hence, left spectral factor balancing and right spectral factor balancing can be interpreted as phase matching reduction algorithms. For positive real SISO systems the phase will be in the interval $\left[-90^{\circ},+90^{\circ}\right]$, hence as long as the phase of the original system is well fitted the positive real property will be preserved.

\subsection{Mixed gramian balancing}

In this section a new combination of gramians is proposed. So far, the only algorithm presented which will guarantee positive real reduced order systems is the
Riccati balancing. When Riccati balancing is used, the system is balanced based on the solution of two Riccati equations. Since the balanced system satisfies the PR equations, this gives positive real reduced order systems. The solution of two Riccati equations is computationally demanding. The idea behind the new approach is to solve one Riccati equation and one Lyapunov equation. As long as one of the PR equations is satisfied, this also holds for the balanced system, and hence for the reduced order system.

By taking the controllability gramian $P$,

$$
A P+P A^{T}+B B^{T}=0
$$

and the available storage gramian $O$,

$$
A^{T} O+O A+\left(O B-C^{T}\right)\left(D+D^{T}\right)^{-1}\left(B^{T} O-C\right)=0
$$

and balancing the system $G(s)$ by using $(P, O)$, we obtain a positive real reduced order system. A similar result can be obtained if the pair $(R, Q)$, consisting of the required supply gramian and the observability gramian, is balanced:

$$
\begin{array}{r}
A R+R A^{T}+\left(R C^{T}-B\right)\left(D+D^{T}\right)^{-1}\left(C R-B^{T}\right)=0 \\
A^{T} Q+Q A+C^{T} C=0 .
\end{array}
$$

Definition 3 The positive real minimal system $G(s)$ is called mixed gramian balanced if,

$$
P=O=\Sigma=\operatorname{diag}\left(\sigma_{1} I_{m 1}, \ldots, \sigma_{q} I_{m q}\right),
$$

or

$$
R=Q=\Sigma=\operatorname{diag}\left(\sigma_{1} I_{m 1}, \ldots, \sigma_{q} I_{m q}\right),
$$

where $\sigma_{1}>\sigma_{2}>\ldots>\sigma_{q}>0$ and $m_{i}$ where $i=(1, \ldots, q)$ are the multiplicities of $\sigma_{i}$ and $m_{1}+\ldots+m_{q}=n$.

The following theorem can now be stated.

Theorem 2 Let the positive real and minimal system $G(s)$ have the mixed gramian balanced realization,

$$
G(s)=\left[\begin{array}{c|c}
A & B \\
\hline C & D
\end{array}\right]=\left[\begin{array}{cc|c}
A_{11} & A_{12} & B_{1} \\
A_{21} & A_{22} & B_{2} \\
\hline C_{1} & C_{2} & D
\end{array}\right],
$$

where,

$$
P=O=\Sigma=\operatorname{diag}\left(\Sigma_{1}, \Sigma_{2}\right),
$$

or

$$
R=Q=\Sigma=\operatorname{diag}\left(\Sigma_{1}, \Sigma_{2}\right),
$$

with

$$
\begin{aligned}
& \Sigma_{1}=\operatorname{diag}\left(\sigma_{1} I_{m 1}, \ldots, \sigma_{k} I_{m k}\right), \\
& \Sigma_{2}=\operatorname{diag}\left(\sigma_{k+1} I_{m_{k+1}}, \ldots, \sigma_{q} I_{q}\right) .
\end{aligned}
$$


Then the reduced order model,

$$
G_{r}(s)=\left[\begin{array}{cc}
A_{11} & B_{1} \\
C_{1} & D
\end{array}\right],
$$

obtained by truncation is positive real.

Proof. We first give a proof for the pair $(P, O)$. Since the system $(A, B, C, D)$ is balanced, the two gramians $(P, O)$ are equal and diagonal, $P=O=\Sigma$, and satisfy one Lyapunov equation and one Riccati equation,

$$
\begin{aligned}
A \Sigma+\Sigma A^{T}+B B^{T} & =0, \\
A^{T} \Sigma+\Sigma A+\left(\Sigma B-C^{T}\right)\left(D+D^{T}\right)^{-1}\left(B^{T} \Sigma-C\right) & =0 .
\end{aligned}
$$

Writing out the second equation in terms of its partitioned matrices gives the following $(1,1)$ block,

$A_{11}^{T} \Sigma_{1}+\Sigma_{1} A_{11}+\left(\Sigma_{1} B_{1}-C_{1}^{T}\right)\left(D+D^{T}\right)^{-1}\left(B_{1}^{T} \Sigma_{1}-C_{1}\right)=0$.

Since $\Sigma_{1}>0$ the positive realness of the reduced order system $\left(A_{11}, B_{1}, C_{1}, D\right)$ can be concluded. The same can be shown for the pair $(R, Q)$.

Further it can be shown that the transfer function of the reduced order system $G_{r}(s)=\left(A_{11}, B_{1}, C_{1}, D\right)$ will be the same either if the gramian pair $(P, O)$ or the gramian pair $(R, Q)$ is used as a basis for the mixed gramian balanced truncation algorithm.

The dual of the system $G(s)$ is written,

$$
G^{T}(-s)=\left[\begin{array}{l|l}
A^{T} & C^{T} \\
\hline B^{T} & D^{T}
\end{array}\right]
$$

Substituting the system $G(s)$ with the dual system $G^{T}(-s)$ in the equations (31)-(32) gives the following equations,

$$
\begin{aligned}
A^{T} P+P A+C^{T} C & =0, \\
A O+O A^{T}+\left(O C^{T}-B\right)\left(D+D^{T}\right)^{-1}\left(C O-B^{T}\right) & =0 .
\end{aligned}
$$

Solving for these equations, where the original system $G(s)$ has been substituted with its dual, $G^{T}(-s)$, is the same as solving for the required supply gramian (33) and the observability gramian (34) of the original system $G(s)$.

Subsequently, $G(s)$ is replaced with its dual $G^{T}(-s)$ in the equations (33)-(34),

$$
\begin{aligned}
A^{T} R+R A+\left(R B-C^{T}\right)\left(D^{T}+D\right)^{-1}\left(B^{T} R-C\right) & =0, \\
A Q+Q A^{T}+B B^{T} & =0 .
\end{aligned}
$$

Solving for these equations, is the same as solving for the controllability gramian and the available storage gramian of the original system $G(s)$.

Due to this duality, which comes from the duality of the Lyapunov equations and the Riccati equations, the transfer function of the balanced system
$G(s)=(A, B, C, D)$ from either using $(P, O)$ or $(R, Q)$ as a basis for the balanced truncation will be zero-state equivalent. When the systems are reduced, due to the duality, the transfer function $G_{r}(s)=\left(A_{11}, B_{1}, C_{1}, D\right)$ will also be zero-state equivalent.

\subsection{Numerical Example}

In this section the different balancing schemes presented in the previous section will be compared in terms of computational time and accuracy. A positive real SISO system of order 350 is given and 8 different truncated systems are first constructed, respectively of orders 350, 300, 250, 200, 150, 100, 50 and 10. All these systems are reduced further to a system of order 2 by using the different balancing schemes presented in the previous sections. The computations are done on a Dell Latitude D800 Notebook with Intel Centrino processor $2 \mathrm{GHz}$ and $1 \mathrm{~GB}$ RAM running MATLAB under Windows XP. The computing times are shown in Figure 2.

As one can see from the figure the Lyapunov balancing is the most efficient in terms of time. This is expected since it is faster to solve Lyapunov equations compared to Riccati equations, since the solution of Riccati equations involves matrix inversions. The mixed gramian balancing lies between the Riccati and Lyapunov balancing in computational time, this is expected since one Lyapunov equation and one Riccati equation is solved. In this example the left and right spectral factor balancing schemes uses approximately the same time as the Riccati balancing. The left and right spectral factor balancing are expected to use more time than the mixed gramian balancing. Even though these algorithms also balances the solution of one Lyapunov and one Riccati equation, more equations are to be solved than for the mixed gramian balancing scheme. The errors between the system of order $n=10$ and the reduced order system of $n=2$ is given in Table 3 . The Bode plot of the original system and the different reduced order systems are shown in Figure 3. The

Table 3: $\|\cdot\|_{\infty}$-error between original and reduced order system of order $n=10$ and $n=2$.

\begin{tabular}{l|c} 
Balancing scheme: & $\left\|G(s)-G_{r}(s)\right\|_{\infty}$ \\
\hline Lyapunov & $9.08 \cdot 10^{5}$ \\
Riccati & $3.53 \cdot 10^{7}$ \\
Mixed gramian & $2.71 \cdot 10^{6}$ \\
Left/Right spectral factor & $2.42 \cdot 10^{6}$ \\
\hline
\end{tabular}

Lyapunov balancing has the best error-performance, but looking at the Bode plot one can see that the re- 
Reduction from order $n_{i}$ to order 2 for different systems

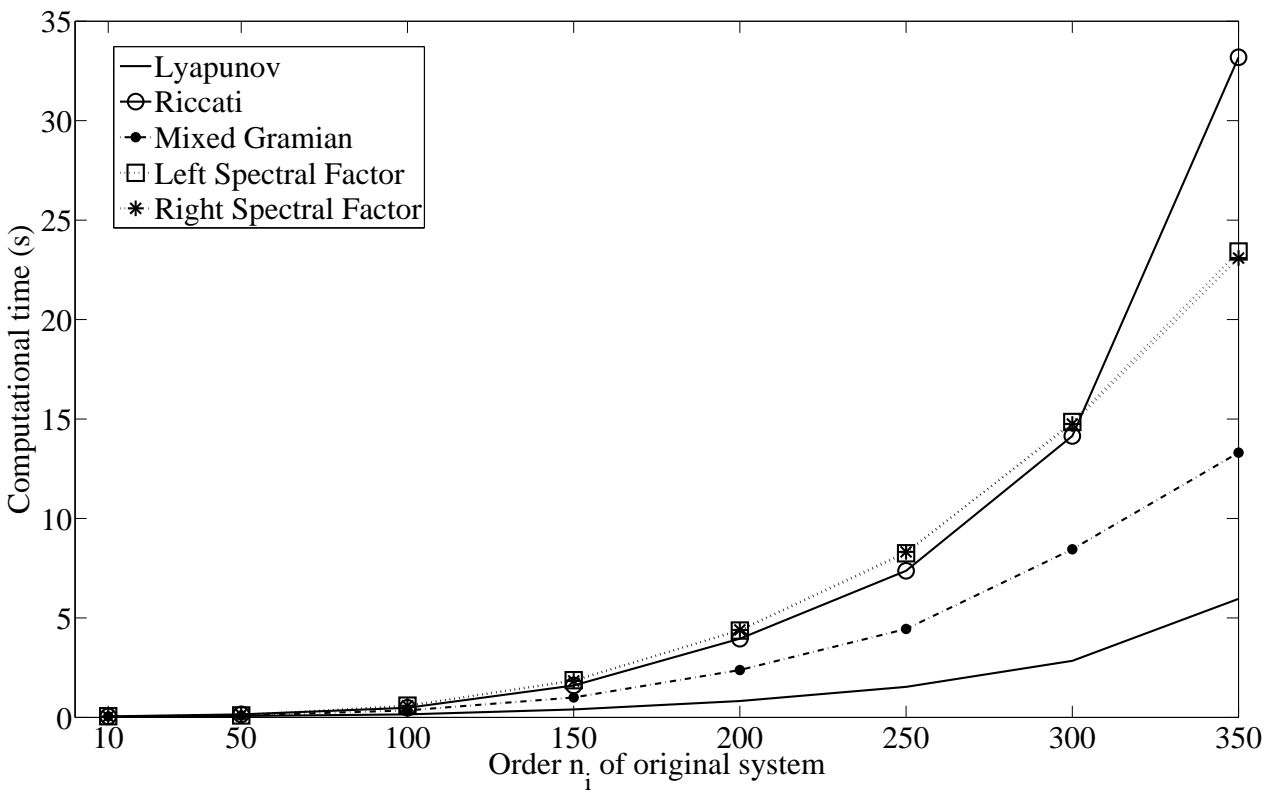

Figure 2: Computational time when reducing the original system of order $\mathrm{n}_{\mathrm{i}}$ down to order 2 for the different balancing schemes.

duced order system is not passive since the phase of the reduced order system exceeds $90 \mathrm{deg}$. The reduced order system using left/right spectral factor balancing is passive, even though this might not be the case, and has the best error performance for this system. The only algorithms which guarantees positive real reduced order systems are the Riccati and mixed gramian balancing. In this example, the mixed gramian balancing can compete with the Riccati balancing both in terms of computational time and reduction error.

\section{Extending Balanced Truncation}

Stable systems can be characterized by the solution of a Lyapunov equation and positive real systems can be characterized by the solution of the positive real lemma. In this section we show that as long as one of the gramians in the balanced truncation scheme satisfies one of these equations the reduced order system generated will be stable or positive real.

\subsection{Stable Projection}

A stable system is characterized by the following theorem (Antoulas, 2005),

Theorem 3 A matrix A is Hurwitz; that is $\operatorname{Re}\left(\lambda_{i}\right)<0$ for all eigenvalues of $A$, if and and only if for any given positive definite symmetric matrix $Q$ there exists a positive definite symmetric matrix $P$ that satisfies the Lyapunov equation,

$$
A P+P A^{T}=-Q .
$$

Moreover if $A$ is Hurwitz, then $P$ is the unique solution of (35).

Let $Y=Y^{T}>0$ be an arbitrary positive real matrix, and $P=P^{T}>0$ be the solution to (35). By substituting $(M, N)$ by the pair $(P, Y)$ in the balanced truncation algorithm in Table 1, it can be shown that the reduced order system will be stable.

Theorem 4 Let the stable and minimal system $G(s)$ have the balanced realization,

$$
G(s)=\left[\begin{array}{c|c}
A & B \\
\hline C & D
\end{array}\right]=\left[\begin{array}{cc|c}
A_{11} & A_{12} & B_{1} \\
A_{21} & A_{22} & B_{2} \\
\hline C_{1} & C_{2} & D
\end{array}\right]
$$

with $P=Y=\Sigma=\operatorname{diag}\left(\Sigma_{1}, \Sigma_{2}\right)$, where the pair $(P, Y)$ comes from,

$$
\begin{array}{r}
\operatorname{arbitrary} Y=Y^{T}>0, \\
A P+P A^{T}+Q=0, \\
P=P^{T}>0 \\
Q=Q^{T}>0 .
\end{array}
$$


Lyapunov Balancing

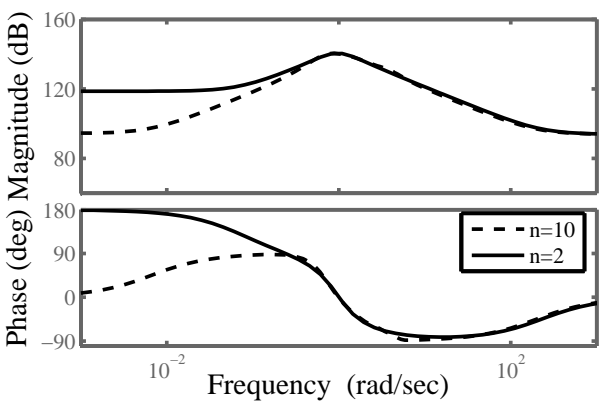

Mixed Gramian Balancing

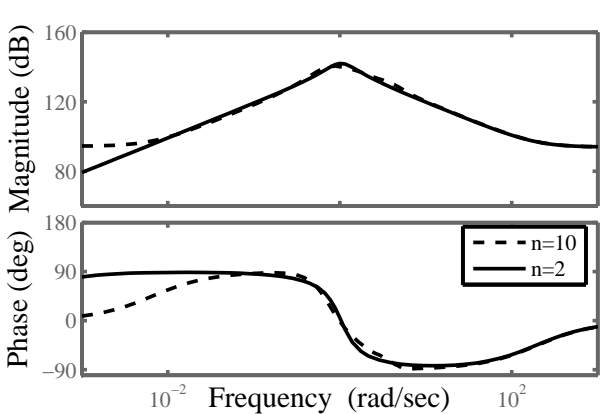

Riccati Balancing

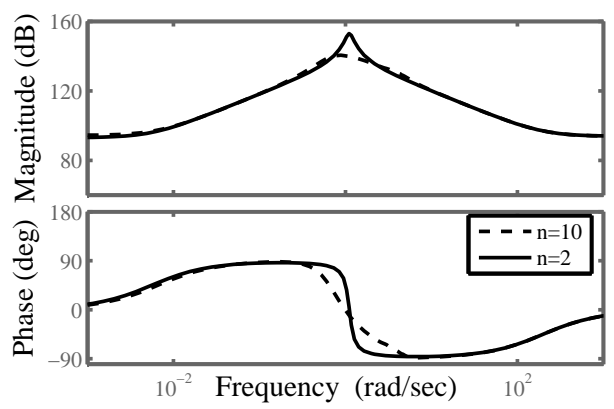

Left $=$ Right Spectral Factor Balancing

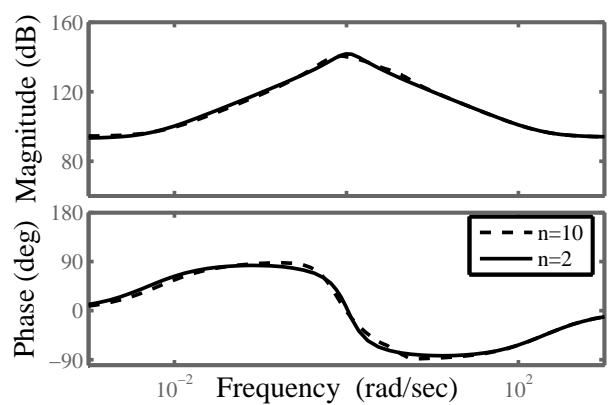

Figure 3: Bode plot of the difference between the original system of order $n=10$ and reduced order system of order $n=2$ using the different balancing schemes. 
Then the associated state transformation $T$ will be a stable state transformation and the reduced order model obtained by truncation is stable.

Proof. Since $(A, B, C, D)$ is balanced, the two gramians $(P, Y)$ are equal and diagonal $P=Y=\Sigma$, and satisfy the following equations,

$$
\begin{array}{r}
P=Y=\Sigma=\left[\begin{array}{cc}
\Sigma_{1} & 0 \\
0 & \Sigma_{2}
\end{array}\right]>0, \\
A \Sigma+\Sigma A^{T}+Q=0 .
\end{array}
$$

Writing out the latter equation in terms of its partitioned blocks gives,

$$
\left[\begin{array}{cc}
A_{11} \Sigma_{1}+\Sigma_{1} A_{11}^{T}+Q_{11} & A_{12} \Sigma_{2}+\Sigma_{1} A_{21}^{T}+Q_{12} \\
A_{21} \Sigma_{1}+\Sigma_{1} A_{11}^{T}+Q_{21} & A_{22} \Sigma_{2}+\Sigma_{2} A_{22}^{T}+Q_{22}
\end{array}\right]=0
$$

For the truncated system $\left(A_{11}, B_{1}, C_{1}, D\right)$ it follows that,

$$
\begin{aligned}
\Sigma_{1}=\Sigma_{1}^{T}>0, \\
Q_{11}=Q_{11}^{T}>0, \\
A_{11} \Sigma_{1}+\Sigma_{1} A_{11}^{T}+Q_{11}=0,
\end{aligned}
$$

and stability of $G_{r}(s)$ can be concluded.

In the next section this result will be extended to positive real systems, and used as a tool for finding new types of algorithms.

\subsection{Positive Real Projection}

In this section we extend the results of Section 2.3. As long as one of the gramians in the balanced truncation algorithm satisfies the PR or DPR equations this will give positive real state transformations. Hence, only one of the gramians in the balanced truncation algorithm must satisfy the positive real lemma.

The positive real system $G(s)=(A, B, C, D)$ will satisfy the positive real equations,

$$
\begin{aligned}
A R+R A^{T} & =-B_{l} B_{l}^{T}, \\
R C^{T}-B & =-B_{l} D_{l}^{T}, \\
-D-D^{T} & =-D_{l} D_{l}^{T},
\end{aligned}
$$

where $R=R^{T}>0, B_{l}$ and $D_{l}$ are to be solved for. These equations can be rewritten as a linear matrix inequality (LMI),

$$
\left[\begin{array}{cc}
A R+R A^{T} & R C^{T}-B \\
C R-B^{T} & -D-D^{T}
\end{array}\right]=-\left[\begin{array}{c}
B_{l} \\
D_{l}
\end{array}\right]\left[\begin{array}{ll}
B_{l}^{T} & D_{l}^{T}
\end{array}\right] \leq 0,
$$

where $R$ is to be solved for. Having $R$ and balancing this with an arbitrary positive real symmetric matrix
$Y, R=Y=\Sigma$ will give a positive real state transformation $T$. Associated with the positive real system $G(s)$ is also a pair of dual positive real equations,

$$
\begin{gathered}
A^{T} O+O A=-C_{r}^{T} C_{r} \\
O B-C^{T}=-C_{r}^{T} D_{r} \\
-D-D^{T}=-D_{r}^{T} D_{r}
\end{gathered}
$$

where $O=O^{T}>0, C_{r}$ and $D_{r}$ are to be solved for. The LMI representation of these equations is,

$\left[\begin{array}{cc}A^{T} O+O A & O B-C^{T} \\ B^{T} O-C & -D-D^{T}\end{array}\right]=-\left[\begin{array}{c}C_{r}^{T} \\ D_{r}^{T}\end{array}\right]\left[\begin{array}{ll}C_{r} & D_{r}\end{array}\right] \leq 0$.

Balancing $O$ with an arbitrary symmetric positive real matrix $Y$ will also give positive real reduced order systems.

Theorem 5 Let the positive real and minimal system $G(s)$ have the balanced realization,

$$
G(s)=\left[\begin{array}{c|c}
A & B \\
\hline C & D
\end{array}\right]=\left[\begin{array}{cc|c}
A_{11} & A_{12} & B_{1} \\
A_{21} & A_{22} & B_{2} \\
\hline C_{1} & C_{2} & D
\end{array}\right],
$$

where,

$$
\begin{array}{r}
R=Y=\Sigma, \\
\text { arbitrary } Y=Y^{T}>0, \\
{\left[\begin{array}{cc}
A R+R A^{T} & R C^{T}-B \\
C R-B^{T} & -D-D^{T}
\end{array}\right] \leq 0} \\
R=R^{T}>0 .
\end{array}
$$

or,

$$
\begin{array}{r}
O=Y=\Sigma, \\
\text { arbitrary } Y=Y^{T}>0 \\
{\left[\begin{array}{cc}
A^{T} O+O A & O B-C^{T} \\
B^{T} O-C & -D-D^{T}
\end{array}\right] \leq 0,} \\
O=O^{T}>0 .
\end{array}
$$

Then the reduced order model,

$$
G_{r}(s)=\left[\begin{array}{cc}
A_{11} & B_{1} \\
C_{1} & D
\end{array}\right]
$$

obtained by truncation is positive real.

Proof. Since $(A, B, C, D)$ is balanced, the two gramians $(R, Y)$ are equal $R=Y=\Sigma$, and satisfy the following equations,

$$
\begin{aligned}
& \Sigma=\Sigma^{T}=\left[\begin{array}{cc}
\Sigma_{1} & 0 \\
0 & \Sigma_{2}
\end{array}\right]>0, \\
& {\left[\begin{array}{cc}
A \Sigma+\Sigma A^{T} & \Sigma C^{T}-B \\
C \Sigma-B^{T} & -D-D^{T}
\end{array}\right] \leq 0 .}
\end{aligned}
$$


Writing out the last equation in terms of its partitioned blocks gives,

$$
\left[\begin{array}{ccc}
A_{11} \Sigma_{1}+\Sigma_{1} A_{11}^{T} & A_{12} \Sigma_{2}+\Sigma_{1} A_{21}^{T} & \Sigma_{1} C_{1}^{T}-B_{1} \\
A_{21} \Sigma_{1}+\Sigma_{2} A_{12}^{T} & A_{22} \Sigma_{2}+\Sigma_{2} A_{22}^{T} & \Sigma_{2} C_{2}^{T}-B_{2} \\
C_{1} \Sigma_{1}-B_{1}^{T} & C_{2} \Sigma_{2}-B_{2}^{T} & -D-D^{T}
\end{array}\right] \leq 0 .
$$

For the system $\left(A_{11}, B_{1}, C_{1}, D\right)$ we then have,

$$
\begin{array}{r}
\Sigma_{1}=\Sigma_{1}^{T}>0, \\
{\left[\begin{array}{cc}
A_{11} \Sigma_{1}+\Sigma_{1} A_{11}^{T} & \Sigma_{1} C_{1}^{T}-B_{1} \\
C_{1} \Sigma_{1}-B_{1}^{T} & -D-D^{T}
\end{array}\right] \leq 0,}
\end{array}
$$

and positive realness of $G_{r}(s)$ can be concluded. The same can be shown for the pair $(O, Y)$.

By knowing these properties, it is now possible to develop new positive real balanced truncation methods. In the next section this will be illustrated by using these properties to develop an algorithm for positive real frequency weighted truncation.

\subsection{Frequency weighted truncation}

The balancing methods in Section (2.1)-(2.3) approximate the system $G(s)$ over all frequencies. For some systems it might be of interest to do an approximation only in a certain range of frequencies. This is called frequency weighted truncation. This can be done by weighting the error system by an input weight $W_{i}(s)$ or/and an output weight $W_{o}(s)$,

$$
\left\|W_{o}(s)\left(G(s)-G_{r}(s)\right) W_{i}(s)\right\|_{\infty},
$$

such that the weighted error is small. Most methods for frequency weighted reduction have focused on keeping the stability properties of the system, a good overview over different methods is given in (Obinata and Anderson, 2001; Antoulas, 2005). In this section we will extend the frequency weighting to positive real input weighted balancing and positive real output weighted balancing.

Let the positive real minimal system $G(s)$ be written as,

$$
G(s)=\left[\begin{array}{ll}
A & B \\
C & D
\end{array}\right],
$$

and let the input weight weight be denoted with $W_{i}(s)$ where,

$$
W_{i}(s)=\left[\begin{array}{cc}
A_{i} & B_{i} \\
C_{i} & D_{i}
\end{array}\right]
$$

The augmented system can now be written as,

$$
\bar{G}(s)=G(s) W_{i}(s)=\left[\begin{array}{cc}
\bar{A}_{i} & \bar{B}_{i} \\
\bar{C}_{i} & \bar{D}_{i}
\end{array}\right]=\left[\begin{array}{cc|c}
A & B C_{i} & B D_{i} \\
0 & A_{i} & B_{i} \\
\hline C & D C_{i} & D_{i} D
\end{array}\right],
$$

this gives the following weighted error system,

$$
\left\|\left(G(s)-G_{r}(s)\right) W_{i}(s)\right\|_{\infty} .
$$

Assuming that $W_{i}(s)$ is chosen such that the overall system $\bar{G}(s)$ is stable, the controllability gramian for the overall system can be solved for,

$$
\bar{A}_{i} \bar{P}+\bar{P} \bar{A}_{i}^{T}+\bar{B}_{i} \bar{B}_{i}^{T}=0,
$$

where $\bar{P}=\bar{P}^{T}>0$ and,

$$
\bar{P}=\left[\begin{array}{cc}
P & \bar{P}_{12} \\
\bar{P}_{21} & \bar{P}_{22}
\end{array}\right]
$$

Expanding (40) gives the following $(1,1)$ block,

$$
A P+P A^{T}+B D_{i} D_{i}^{T} B^{T}=0,
$$

where $P=P^{T}>0$. Further, the available storage gramian is solved for the unweighted system (38),

$$
A^{T} O+O A+\left(O B-C^{T}\right)\left(D+D^{T}\right)^{-1}\left(B^{T} O-C\right)=0 .
$$

By combining $(P, O)$ from (41) and (42) input weighted positive real reduced order systems can be obtained. Assuming that $(P, O)$ are balanced, $P=O=\Sigma$, we have the following equations,

$$
\begin{aligned}
\Sigma=\Sigma^{T}=\left[\begin{array}{cc}
\Sigma_{1} & 0 \\
0 & \Sigma_{2}
\end{array}\right] & >0, \\
A \Sigma+\Sigma A^{T}+B D_{i} D_{i}^{T} B^{T} & =0, \\
A^{T} \Sigma+\Sigma A+\left(\Sigma B-C^{T}\right)\left(D+D^{T}\right)^{-1}\left(B^{T} \Sigma-C\right) & =0 .
\end{aligned}
$$

Writing down the $(1,1)$ blocks of the two latter equations gives,

$$
A_{11} \Sigma_{1}+\Sigma_{1} A_{11}^{T}+B_{1} D_{i} D_{i}^{T} B_{1}^{T}=0
$$

$A_{11}^{T} \Sigma_{1}+\Sigma_{1} A_{11}+\left(\Sigma_{1} B_{1}-C_{1}^{T}\right)\left(D+D^{T}\right)^{-1}\left(B_{1}^{T} \Sigma_{1}-C_{1}\right)=0$,

where one can see that the reduced order system satisfies the Riccati equation and hence it will be positive real. By choosing the input weight $W_{i}(s)$ properly, the weighted error in (39) will be small.

This approach can also be used on output weighted systems. Let the system's output weight be denoted by $W_{o}(s)$, where,

$$
W_{o}(s)=\left[\begin{array}{cc}
A_{o} & B_{o} \\
C_{o} & D_{o}
\end{array}\right]
$$

The augmented output weighted system can now be written as,

$\hat{G}(s)=W_{o}(s) G(s)=\left[\begin{array}{ll}\hat{A}_{o} & \hat{B}_{o} \\ \hat{C}_{o} & \hat{D}_{o}\end{array}\right]=\left[\begin{array}{cc|c}A & O & B \\ B_{o} C & A_{o} & B_{o} D \\ \hline D_{o} C & C_{o} & D_{o} D\end{array}\right]$, 
with the associated weighted error system,

$$
\left\|W_{o}(s)\left(G(s)-G_{r}(s)\right)\right\|_{\infty} .
$$

Assuming that $W_{o}(s)$ is chosen such that the overall system $\hat{G}(s)$ is stable, then the observability gramian for the overall system can be solved for,

$$
\hat{A}_{o}^{T} \hat{Q}+\hat{Q} \hat{A}_{o}+\hat{C}_{o}^{T} \hat{C}_{o}=0,
$$

where $\hat{Q}=\hat{Q}^{T}>0$. Let $\hat{Q}$ be written as,

$$
\hat{Q}=\left[\begin{array}{cc}
Q & \hat{Q}_{12} \\
\hat{Q}_{21} & \hat{Q}_{22}
\end{array}\right] \text {. }
$$

Expanding (43) gives the following $(1,1)$ block,

$$
A^{T} Q+Q A+C^{T} D_{o}^{T} D_{o} C=0,
$$

where $Q=Q^{T}>0$. Further, the required supply gramian is solved for the unweighted system (38),

$$
A R+R A^{T}+\left(R C^{T}-B\right)\left(D+D^{T}\right)^{-1}\left(C R-B^{T}\right)=0 .
$$

By combining $(R, Q)$ from (44) and (45) output weighted positive real reduced order systems can be obtained. When $(R, Q)$ are balanced, $R=Q=\Sigma$, we have the following equations,

$$
\begin{aligned}
\Sigma=\Sigma^{T}=\left[\begin{array}{cc}
\Sigma_{1} & 0 \\
0 & \Sigma_{2}
\end{array}\right] & >0, \\
A \Sigma+\Sigma A^{T}+C^{T} D_{o}^{T} D_{o} C & =0, \\
A \Sigma+\Sigma A^{T}+\left(\Sigma C^{T}-B\right)\left(D+D^{T}\right)^{-1}\left(C \Sigma-B^{T}\right) & =0 .
\end{aligned}
$$

By writing down the $(1,1)$ blocks of the two latter equations,

$$
A_{11} \Sigma_{1}+\Sigma_{1} A_{11}^{T}+C_{1}^{T} D_{o}^{T} D_{o} C_{1}=0
$$

$A_{11} \Sigma_{1}+\Sigma_{1} A_{11}^{T}+\left(\Sigma_{1} C_{1}^{T}-B_{1}\right)\left(D+D^{T}\right)^{-1}\left(C_{1} \Sigma_{1}-B_{1}^{T}\right)=0$,

one can see that the reduced order system satisfies a Riccati equation, and hence it will be positive real.

\section{Concluding Remarks}

A survey of Lyapunov balancing and stochastic balancing have been given. Based on these algorithms a new approach for obtaining positive real balanced truncation has been suggested, which we called mixed gramian balancing. This approach is a combination of Lyapunov balancing and Riccati balancing, and relies on balancing the solution of one Lyapunov and one Riccati equation. Riccati balancing relies on the solution of two Riccati equations, while the mixed balancing relies on the solution of one Lyapunov equation and one Riccati equation. This is less computationally demanding.

Further it has been shown that in order to obtain positive real truncated systems, only one of the gramians in the balancing algorithm need to satisfy either the PR or the DPR equations. This opens for new combinations of gramians in order to obtain positive real truncated systems. Here it has been used to obtain positive real frequency weighted truncation. For future research it would be of interest to find error bounds for the proposed algorithms.

In the literature there already exists model reduction methods for the $H_{2}$ or $H_{\infty}$ norm which give stable systems (Yan and Lam, 1999). For future work it would be interesting to see if it is possible to extend some of this work to positive real system exploiting the properties in this paper.

\section{Acknowledgments}

This project was partially supported by the Centre for Ships and Ocean Structures (CeSOS) at the Norwegian University of Science and Technology (NTNU), through the Research Council of Norway. The second author was also supported by NSF contract ACI-0324944, and by the Belgian Network DYSCO (Dynamical Systems, Control, and Optimization), funded by the Interuniversity Attraction Poles Programme, initiated by the Belgian State, Science Policy Office.

\section{References}

Antoulas, A. Approximation of Large-Scale Dynamical Systems. SIAM, 2005.

Benner, P., Mehrmann, V., and Sorensen, D. Dimension Reduction of Large-Scale Systems. Springer, 2005 .

Chen, C.-T. Linear System Theory and Design. Oxford University Press, 1999.

Desai, U. and Pal, D. A transformation approach to stochastic model reduction. IEEE Transactions on Automatic Control, 1984. AC-29:1097-1100.

Glover, K. All optimal Hankel-norm approximations of linear multivariable systems and their $L^{\infty}$-error bounds. International Journal of Control, 1984. 39:1115-1193.

Green, M. Balanced Stochastic Realizations. Linear Algebra and its Applications, 1988. 98:211-247. 
Gugercin, S. and Antoulas, A. A survey of model reduction and some new results. International Journal of Control, 2004. 77:748-766.

Harshavardhana, P., Jonckheere, E., and Silverman, L. Stochastic balancing and approximation-stability and minimality. IEEE Transaction on Automatic Control, 1984. 29:744-746.

Moore, B. Principal component analysis in linear systems: Controllability, observability, and model reduction. IEEE Transactions on Automatic Control, 1981. 23:17-32.

Mullis, C. and Roberts, R. Synthesis of minimum roundoff noise fixed point digital filters. IEEE Transactions on Circuits and Systems, 1976. CAS-23:551562 .

Obinata, G. and Anderson, B. Model Reduction for Control System Design. Springer, 2001.

Opdenacker, P. and Jonckheere, E. A state space approach to approximation by phase matching. Modelling, Identification and Robust Control, 1986. page 1986.

Phillips, J., Daniel, L., and Silveira, L. Guaranteed passive balancing transformations for model order reduction. IEEE Transactions on Computer-Aided Design of Integrated Circuits and Systems, 2003. 22:1027-1041.

Willems, J. Dissipative dynamical systems, Part I: General Theory. Arch. Rat. Mech. An., 1971. 45:321-351.

Yan, W.-Y. and Lam, J. An approximate approach to $\mathrm{H}^{2}$ optimal model reduction. IEEE Transaction on Automatic Control, 1999. 44:1341-1358. 